\title{
COMMISSION 47: COSMOLOGY
} (COSMOLOGIE)

\author{
PRESIDENT: J.A. Peacock \\ VICE-PRESIDENT: S.J. Lilly \\ ORGANIZING COMMITTEE: G. Bruzual, L. Campusano, Y. Chu, \\ L. DaCosta, J. Einasto, G. Ellis, D. Koo, O. Le Fèvre, J. Narlikar, P. Shaver, \\ Y. Suto, A. Szalay, R. Webster, S. White \& A. Wolfe
}

\section{Introduction}

The past three years have been an astonishing period of progress in cosmology. A number of major developments have virtually completed the establishment of a consensus 'standard model' for the subject. A good approximation to the real universe appears to be given by a flat model with density parameters of $(0.7,0.25,0.05)$ in respectively vacuum energy, cold dark matter, and baryons. This would have been regarded as highly controversial in the mid 1990 s, but is now generally adopted as the default model, where for good measure it is generally accepted that the Hubble constant is within $10 \%$ of $70 \mathrm{~km} \mathrm{~s}^{-1}$. One should of course worry about the possibility of a herd instinct, but this picture is buttressed by an impressive number of independent tests, and some of these are summarized below.

\section{The Distance Scale}

A central aim in cosmology is the testing of cosmological models via the direct measurement of the distance-redshift relation. This aim is now becoming a reality, thanks in the main to the possibility of using SNe Ia as accurate standard candles. These are the most important of the distance indicators that are used to carry the Cepheid distance scale out to galaxies where perturbations to the Hubble flow are negligible. The HST key programme has now completed its measurements of Cepheids. Combining these with SNe data and other indicators, the conclusion is that the dimensionless Hubble parameter is $h=0.72 \pm 10 \%$ (Freedman et al. 2001). The dominant remaining source of uncertainty is now the distance to the LMC, where the Cepheid scale is anchored.

Moving beyond the first time derivative of the scale factor, the SNe Hubble diagram continues to provide strong evidence for a negative-pressure component to the cosmic energy budget, leading to an expansion that fails to decelerate at present (Riess 2000). The prediction of such vacuum-dominated models is that they should display deceleration at high redshift, since the vacuum energy will be subdominant at such times. The first evidence for this effect appears to have been seen, via the serendipitous discovery of a SN at $z=1.7$ (Riess et al. 2001).

\section{Galaxy Clustering}

The outstanding recent studies of large-scale structure have been the 2dF Galaxy Redshift Survey (Colless et al. 2001) and the Sloan Digital Sky Survey (Stoughton et al. 2002). The former has completed its observational phase, yielding some 220,000 redshifts; the latter is still underway. The key scientific results from the analysis of the 2dFGRS include the measurement of the galaxy luminosity function and its dependence on galaxy type (Madgwick et al. 2002); the demonstration that galaxy clustering is an increasing function 
of luminosity for all galaxy types (Norberg et al. 2001, 2002); the first detailed detection of redshift-space clustering distortions from peculiar velocities (Peacock et al. 2001); the measurement of the galaxy power spectrum (Percival et al. 2001). The power spectrum is especially important: in combination with CMB data, it allows the cosmological model to be measured very accurately. The favoured model is a flat universe dominated by very nearly scale-invariant scalar fluctuations, with $\Omega_{m}=0.31 \pm 18 \%$ and $h=0.67 \pm 7 \%$ (Efstathiou et al. 2001; Percival et al. 2002). Interestingly, the galaxy distribution seems to display a rather small degree of bias with respect to the mass distribution (Lahav et al. 2002; Verde et al. 2002).

\section{The Microwave Background}

The year 1992 was one of the most significant in the history of cosmology, witnessing the COBE detection of the anisotropy in the cosmic microwave background. The recent developments in the CMB have been no less revolutionary. A large number of groups have performed accurate measurements of the CMB with resolution down to the arcminute scale (e.g. Boomerang: de Bernardis et al. 2002; Maxima: Lee et al. 2001; DASI: Pryke et al. 2002; VSA: Scott et al. 2002; CBI: Mason et al. 2002). These have shown that the power spectrum of the temperature fluctuations contains power at the harmonics of a fundamental scale of order 1 degree in size. These peaks are the natural prediction of the simplest models of structure formation via gravitational instability, in which the matterradiation fluid undergoes acoustic oscillations.

Hardly had the community had time to digest these remarkable developments, however, than the field moved on to what will surely be the dominant theme of the next few years: CMB polarization. The nature of Thomson scattering means that some polarization of the anisotropies is expected, and this has been convincingly detected by the DASI group (Kovac et al. 2002). This opens the door to detailed exploration of the era of reionization, and the ultimate goal of testing inflation via the detection of tensor mode anisotropies.

\section{Gravitational Lensing}

Gravitational lensing studies have made a number of very significant recent advances. Galaxy-galaxy lensing was detected at high significance in the SDSS data (Fischer et al. 2000 ), allowing measurements of the galaxy-mass correlation function, or (equivalently) the mean mass of the dark haloes surrounding galaxies. This can be studied in some detail, so that variations in halo mass with galaxy properties can be measured, and also the shape of the haloes, which appear to be flattened to an extent that is compatible with the predictions of CDM models.

Lensing has also achieved a major long-term goal, which is to measure the statistical variations in the distortion of background galaxies between randomly-selected fields. Various groups have published data on this phenomenon of cosmic shear, and these are in good agreement with each other (e.g. Bacon et al. 2000; Wittman et al. 2000; Wilson, Kaiser \& Luppino 2001; Van Waerbeke et al. 2001). The variation of shear with scale relates to the matter power spectrum, and the data are in good agreement with the standard $\Omega_{m}=0.3$ $\Lambda$ CDM model. The sensitivity to power spectrum shape is not presently very high, but these studies can in principle give the most accurate determination of the normalization of the mass fluctuations - usually expressed as the parameter $\sigma_{8}$. Values between $\sigma_{8}=0.75$ and 0.95 have derived. The next generation of this work should pin down $\sigma_{8}$ to better than $10 \%$, which will have important implications: the lower values match better with the raw normalization inferred from the $\mathrm{CMB}$, implying that the optical depth to the last-scattering surface cannot be very large. 


\section{High-Redshift Objects}

The outstanding recent development in studies of the high-redshift universe has been the discovery of quasars at up to $z=6.3$ by the SDSS (Fan et al. 2001). At this redshift, the suppression of the Lyman continuum is almost total - well in excess of extrapolation from lower redshifts. This has given rise to speculation that observations are now probing close to the era of cosmological reionization, where the HII regions from the earliest discrete sources percolate for the first time (Becker et al. 2001).

The intergalactic medium is being studied in increasing detail via absorption-line spectroscopy. Deuterium is now measured in the Lyman-alpha forest at high precision. Interpreted with the aid of primordial nucleosynthesis theory, the baryon density is measured accurately to be $\Omega_{b} h^{2}=0.020 \pm 0.001$ (Burles et al. 2001). We are also able to begin studying in detail the interactions between high-redshift galaxies and the intergalactic medium, and thus the process by which heavy elements were spread throughout the universe (Adelberger et al. 2002).

\section{Theoretical Cosmology}

Theoretical cosmology has also made great recent advances. Numerical studies of cosmological structure are now able to capture many features of the real universe. Large-scale distribution of dark matter can be simulated with $10^{9}$ particles to cover the entire visible 'Hubble volume' (Evrard et al. 2002), or study the sub-structure of a single CDM halo with more than $10^{7}$ particles (Ghigna et al. 2000). Simulations with gas are now able to match in great detail the properties of the intergalactic medium at high redshift (e.g. Meiksin, Bryan \& Machacek 2001; Petry et al. 2002). The formation of highly nonlinear structures such as galaxies is a greater challenge, but semi-analytic work is now starting to explain how galaxies are biased with respect to the dark matter, showing that their correlations can certainly be made consistent with the standard CDM picture (Benson et al. 2000).

There is also great activity on the more fundamental side of cosmology. Understanding the negative-pressure vacuum energy is a great challenge. There is some disagreement as to what to call this: regrettably, the term 'dark energy' is gaining ground, but this of course means exactly the same as 'dark matter'. Another alternative is 'quintessence', but this is perhaps best reserved for the more specific case where the vacuum energy comes from a scalar field rolling down a potential, in such a way that the effective cosmological constant only switches on at late times (Zlatev et al. 1999; Peebles \& Ratra 2002). This would be an attractive model, except that the energy scale in the potential has to be chosen to make the transition happen now. Thus, quintessence models do not solve the 'coincidence problem'; furthermore, they involve potentials in which the energy density asymptotically tends to zero, for which there is no compelling rationale. Therefore, at present it seems more likely that the correct explanation of the vacuum energy is anthropic (Vilenkin 2001), and that all attempts to measure its equation of state are bound to find $w=p / \rho=-1$.

As summarized above, current CMB data are very consistent with the predications of simple inflationary models - although this is far from a verification of inflation, despite some claims. From this point of view, a very healthy recent development has been the proposal of an alternative means of generating the initial phase of the universe. The 'ekpyrotic universe' (Khoury et al. 2001) uses the 'brane world' concept of the existence of extra dimensions to generate the big bang via brane collisions. Indeed, it has been proposed that such collisions can recur infinitely many times, yielding a cyclic universe of indefinite age (Steinhardt \& Turok 2002). It remains to be seen whether these ideas will generate similar observational features to inflationary models, but exploration of these possibilities is an exciting challenge.

J.A. Peacock

President of the Commission 


\section{References}

Adelberger K. et al., astro-ph/0210314

Bacon D., Refregier A., Ellis R.S., 2000, MNRAS, 318, 625

Becker R.H. et al., 2001 AJ, 122, 2850

Benson A.J. et al., 2000, MNRAS, 311, 793

Burles S., Nollett K.M., Turner M.S., 2001, ApJ, 552, L1

Colless M. et al., 2001, MNRAS, 328, 1039

de Bernardis P. et al., 2002, ApJ, 564, 559

Efstathiou G. et al., 2002, MNRAS, 330, L29

Evrard A.E. et al., 2002, ApJ, 573, 7

Fan X. et al., $2001 \mathrm{AJ}, 122,2833$

Fischer P. et al., 2000, AJ, 120, 1198

Freedman W.L. et al., 2001, ApJ, 553, 47

Ghigna S. et al., 2000, ApJ, 544, 616

Kovac J. et al., 2002, astro-ph/0209478

Khoury J. et al., 2001, Phys. Rev., D64, 123522

Lahav O. et al., 2002, MNRAS, 333, 961

Lee A.T. et al., 2001, ApJ, 561, L1

Madgwick D. et al., 2002, MNRAS, 333, 133

Mason B. et al, 2002, astro-ph/0205384

Meiksin A., Bryan G., Machacek M., 2001, MNRAS, 327, 296

Norberg P. et al., 2001, MNRAS, 328, 64

Norberg P. et al., 2002, MNRAS, 332, 827

Peacock J.A. et al., 2001, Nature, 410, 169

Peebles P.J.E., Ratra B., 2002, astro-ph/0207347

Percival W.J. et al., 2001, MNRAS, 327, 1297

Percival W.J. et al., 2002, astro-ph/0206256

Petry C.E. et al., 2002, ApJ, 566, 30

Pryke C. et al., 2002, ApJ, 568, 46

Riess A., 2000, PASP, 112, 1284

Riess A. et al., 2001, ApJ, 560, 49

Scott P. et al., 2002, astro-ph/0205380

Steinhardt P.J., Turok N.J., 2002, Science, 296, 1436

Stoughton C. et al., 2002, AJ, 123, 485

Van Waerbeke L. et al., 2001, A\&A, 374, 757

Verde L. et al., 2002, MNRAS, 335, 432

Wilson G., Kaiser N., Luppino G.A., 2001, ApJ, 556, 601

Wittman D.M., Tyson J.A., Kirkman D., Dell'Antonio I., Bernstein G., 2000, Nature, 405, 143

Vilenkin A., 2001, hep-th/0106083

Zlatev I., Wang L., Steinhardt P.J., 1999, Phys. Rev. Lett., 82, 896 